\title{
An investigation into the surface termination and near-surface bulk doping of oxygen-terminated diamond with lithium at various annealing temperatures
}

\author{
Sami Ullah ${ }^{1,2}$ (1) $\cdot$ Liam Cullingford ${ }^{2,3} \cdot$ Tongfei Zhang $^{2} \cdot$ Jing Ren Wong ${ }^{2} \cdot$ Gary Wan $^{1,2} \cdot$ Mattia Cattelan $^{3} \cdot$ Neil Fox $^{1,3}$
}

Received: 12 December 2020 / Accepted: 10 April 2021 / Published online: 21 April 2021

(c) The Author(s) 2021

\begin{abstract}
An alternative method of doping and surface functionalization of diamond using a chemical route was explored. The interaction of Li with the surface and bulk of oxygen-terminated diamond was investigated using Angle-Resolved X-ray Photoemission Spectroscopy (ARXPS). A stable $\mathrm{LiO}_{2}$ termination of diamond (100) surface and doping of near-surface diamond bulk was achieved up to an annealing temperature of $850^{\circ} \mathrm{C}$. The changes in interaction between the species involved $(\mathrm{C}, \mathrm{O}, \mathrm{Li})$ and their stoichiometric ratios at the surface were investigated as a function of annealing temperature. This was done using ARXPS peak analysis.
\end{abstract}

\section{Introduction}

Our age has seen a huge demand on energy sources to provide for our day-to-day uses which we tend to fulfill by nonrenewable sources. A way of decreasing so much burden on nonrenewable sources of energy is to produce materials with low work function. Using these materials, energy can be produced through thermionic emission where thermal energy makes the material emit electrons and hence provides us with devices that produce electric power. Diamond has been a focus of researchers as a potential material for creating low work function (thermionic emission) devices [1-4]. Tuning of the electrical properties of diamond by controlled doping of elements like oxygen, nitrogen, lithium, sodium, or boron $[5,6]$ has multiplied the interest in diamond research. Li-doped diamond films are believed to be a potential approach to create $n$-type semiconducting diamond with low resistivity and room temperature dopant activation. Theoretical studies predict the potential of $\mathrm{Li}$ to act as a shallow donor [7-9]. The energy gap between the Li donor

Sami Ullah

sami.ullah@bristol.ac.uk

1 School of Physics, HH Wills Physics Laboratory, University of Bristol, Tyndall Avenue, Bristol BS8 1TL, UK

2 Bristol Centre for Functional Nanomaterials, University of Bristol, Tyndall Avenue, Bristol BS8 1TL, UK

3 School of Chemistry, Cantocks Close, Bristol BS8 1TS, UK levels to conduction band of diamond is less than $0.3 \mathrm{eV}$ [10], but effective Li doping is very difficult to obtain. One of the reasons for this is the low solubility of $\mathrm{Li}$ in diamond [11]. Researchers have previously attempted to incorporate $\mathrm{Li}$ into the diamond lattice by the processes of implantation $[12,13]$, diffusion $[14,15]$, and by gas phase in chemical vapor deposition (CVD) [16-18]. Zamir et al. [19] have demonstrated the possibility of doping diamond with higher concentrations of dopants $\mathrm{Li}$ and $\mathrm{N}$ using lithium nitride suspension and gaseous ammonia, respectively. Their method resulted in a higher concentration of both species than those reported by previous diffusion and ion implantation studies, with no lattice damage.

Besides tuning the conductivity of diamond, surface terminations can induce negative electron affinity (NEA) and hence provide ways to tune the work function (WF). It was found that hydrogen termination at the diamond surface induces an NEA of $-1 \mathrm{eV}$ for a number of crystallographic planes [20], this generates a small dipole on the diamond surface leading to a work function of 2.85-3.9 eV [20-22]. However, H-terminated diamond surfaces suffer from the phenomena of water adsorption, leading to charge transfer and unnecessary band bending $[23,24]$. Along with the instability of $\mathrm{H}$ termination at higher temperatures $\left(>700{ }^{\circ} \mathrm{C}\right)[25]$, this restricts the use of $\mathrm{H}$-terminated surface in thermionic applications. Cs-O-terminated diamond surface has been demonstrated to possess a work function of $1.5 \mathrm{eV}$, but Cs desorbs above a temperature of $400{ }^{\circ} \mathrm{C}$ [26]. Also, a work function 
of $1 \mathrm{eV}$ is desirable for low-temperature thermionic applications which leads to a new interest of developing thermally stable, but highly polar surface structures. The study of low WF diamond has resulted in reports of many alternatives to hydrogen which can induce an NEA. This includes thin metal films [27], alkali-metal monolayers [28], alkali-halide films [29, 30], and alkali-oxide monolayers [26].

Oxygenation also plays a role in work function changes, as has been found in the cases of $\mathrm{Si}$ and Ge. Oxygen is also able to modify surface dipoles created by alkalimetal adsorption. In the case of diamond, the lightest alkali-metal, $\mathrm{Li}$, on diamond surfaces has been observed to lower the work function of and at the same time form stronger bonds with the surface than the heavier alkali metals.

This has been demonstrated by O'Donnell [31] who performed the first ab initio calculations for $\mathrm{Li}$ adsorbed onto the $C(100)-2 \times 1$ and $C(100)-1 \times 1$ :O surfaces. The results showed a large NEA and high binding energy per $\mathrm{Li}$ atom for the system consisting of a full monolayer of $\mathrm{Li}$, adsorbed on a fully oxygenated Diamond (100) surface. These results indicate that the properties of Li adsorption on diamond generally agree with those of heavier alkali metals on silicon, diamond, and germanium. O'Donnell demonstrated the formation of a stable $\mathrm{Li}-\mathrm{O}$ termination of diamond by physical vapor deposition of a monolayer of Li on the diamond surface which develops and exhibits NEA even at an elevated temperature of $800{ }^{\circ} \mathrm{C}$ [32].

In this work we are seeking an alternative method of introducing and coordinating $\mathrm{Li}$ with oxygen on the diamond surface. As mentioned earlier, the introduction of $\mathrm{Li}$ atoms into diamond lattice has been demonstrated[19], but here we are exploring the novel and facile chemical means to realize a more controlled approach of creating a stable -O-Li dipole. This will lead to NEA condition uniformity over the diamond surface. We also plan to study and visualize for the first time the effect of temperature on the concentration of $\mathrm{Li}$ atoms in the diamond and on the $\mathrm{Li}-\mathrm{O}$ species on the surface of diamond sample. We are doping oxygen-terminated boron-doped polycrystalline diamond and nitrogen-doped High-Pressure High-Temperature (HPHT) diamond (111) with Li atoms using microwave plasma. The oxygen termination was induced by acid washing. We analyzed the sample with X-Ray Photoemission Spectroscopy (XPS) and Raman spectroscopy. The main aim of this study is to analyze the change in concentration of $\mathrm{Li}$ atoms in the bulk and on the surface of diamond with annealing temperature. This study can reveal an important piece of information about the optimization of surface termination of diamond with the -O-Li group. This is crucial for developing an effective NEA diamond surface for further applications.

\section{Materials and methods}

Boron-doped polycrystalline diamond (BDD) with dimensions of $10 \times 10 \mathrm{~mm}, 0.6 \mathrm{~mm}$ thickness, and HPHT Nitrogen-doped single crystal (111) with dimensions of $3 \times 3 \mathrm{~mm}$ and $0.3 \mathrm{~mm}$ thickness were used as the substrates. The process of nitrogen and boron doping is described elsewhere [19].

The substrates were acid washed with a solution of sulphuric acid $\left(\mathrm{H}_{2} \mathrm{SO}_{4}\right)$ and potassium nitrate $\mathrm{KNO}_{3}$ to remove any metallic contaminants present on the surface, this process also terminated the surfaces with oxygen [33]. The substrates were hydrogen plasma treated in a microwave plasma at $900{ }^{\circ} \mathrm{C}$ for $2 \mathrm{~min}$, followed by $2 \mathrm{~min}$ at $500{ }^{\circ} \mathrm{C}$ as described in [22]. This served to smoothen the diamond surface and has been proven to terminate the sample with hydrogen [34]. Lithium nitride $\left(\mathrm{Li}_{3} \mathrm{~N}\right)$, a source of $\mathrm{Li}$, was obtained commercially as a powder from CERAC (99.5\% purity, $250 \mu \mathrm{m}$ particle size). It has already been established that the stability of $\mathrm{Li}_{3} \mathrm{~N}$ suspension with any oxygen-free liquid increases with an increase in polarity of that liquid, with chloroform being the best of all [19]. So, a stable suspension containing $85 \mathrm{mg}$ of Lithium nitride powder suspended in a solution of $5 \mathrm{mg}$ of polyoxyethylene ether (POE) in $5 \mathrm{ml}$ of chloroform was prepared. It resulted in a reddish-brown suspension which was sonicated for $1 \mathrm{~h}$ in an ultrasonication bath.

The surface quality of both diamond substrates was analyzed with scanning electron microscopy (SEM) and Raman spectroscopy for bulk damage to the $s p^{3}$ character or evidence of strong graphitization. The lithium nitride solution was drop cast on the surface of both substrates at a volume of $10 \mu \mathrm{l}$ using a $\mu$-pipette. The substrates were mounted in the NanoESCA facility and were annealed at different temperatures ranging from 500 to $850{ }^{\circ} \mathrm{C}$ under ultra-high vacuum (UHV) conditions.

XPS measurements were acquired with samples at room temperature (RT) with a monochromatic Al K $\alpha$ source $(1486.7 \mathrm{eV})$ in as-prepared condition and after each annealing step. A pass energy of $20 \mathrm{eV}$ was used, for an overall energy resolution of $600 \mathrm{meV}$. The angle between sample and electron analyser has been set to $45^{\circ}$. After annealing at $750{ }^{\circ} \mathrm{C}$ and $850{ }^{\circ} \mathrm{C}$, additional XPS measurements at more normal angle, i.e., $25^{\circ}$, were acquired, allowing more in-depth analysis of the sample. 
(a)

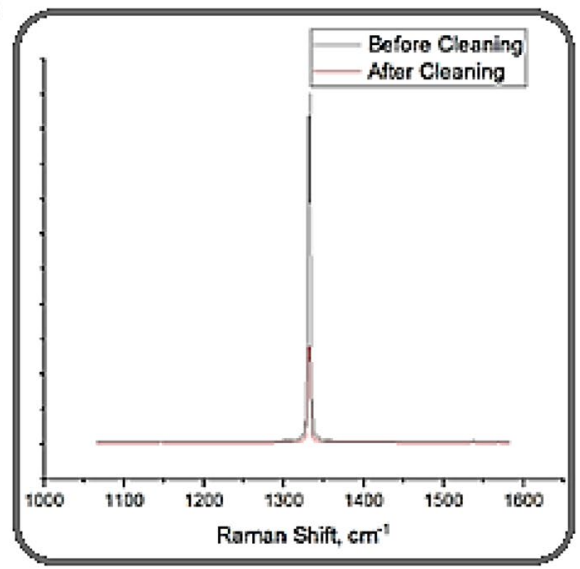

(b)

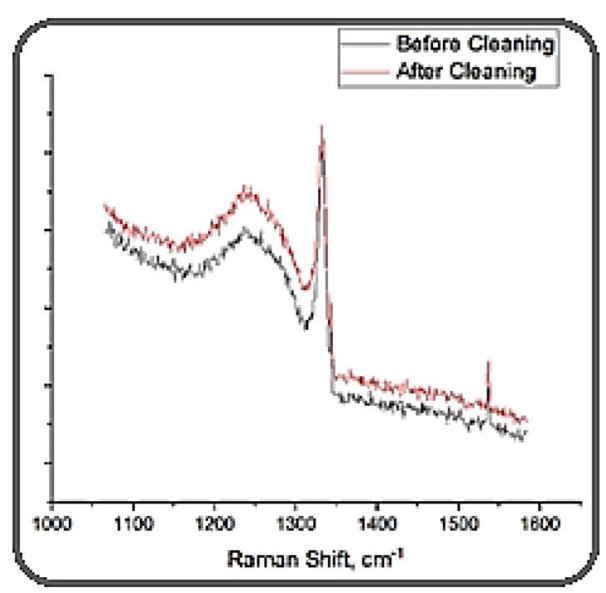

Fig. 1 a Raman spectra of HPHT Single crystal; b Raman spectra of Polycrystalline Diamond after acid washing

\section{Results and discussion}

We investigated any bulk defects and graphitization due to cleaning with Raman spectroscopy (Fig. 1a and b) using a $785 \mathrm{~nm}$ laser. No signs of significant graphitization or loss of $s p^{3}$ character were observed in HPHT diamond; however, there is a small peak around $1540 \mathrm{~cm}^{-1}$, which corresponds to the defect clusters in BDD diamond and could not be removed with acid washing. The acid wash did not create any visible defects while getting rid of the surface impurities and graphitized portions.

XPS gives us qualitative and quantitative information of the sample surface such as percentage composition and oxidation state of surface species. Figure $2 \mathrm{a}$ and $\mathrm{b}$ shows the $\mathrm{O} 1 s$ and $\mathrm{Li} 1 s$ peaks at different annealing temperatures. The deconvolution of the $\mathrm{O} 1 s$ peaks reveals the presence of a mix of ether and carbonyl bonds, while another peak for metal oxide can be seen at a lower binding energy of $530.77 \mathrm{eV}$ which can be attributed to $\mathrm{LiO}_{2}$ [35]. At higher annealing temperatures, we see a decrease in the peak area of $\mathrm{O} 1 s$, indicating loss of oxygen as has been seen on many samples. The metal oxide peak increases in area at $650{ }^{\circ} \mathrm{C}$ and thereafter starts decreasing at higher temperatures. However, some of the oxygen is still retained at $850{ }^{\circ} \mathrm{C}$ which points towards the stability of oxygen-terminated diamond surface as the oxygen doesn't completely escape with metal atoms, but binds to the carbon atoms on the surface of diamond.

A similar trend can be seen in Li $1 s$ peaks where a deconvolution of the peak reveals the presence of ionic $\mathrm{Li}^{2+}$ component along with another low binding energy (B.E.) peak which can be attributed to the metallic Li diffused into the near-surface bulk of diamond. However, this point cannot be proven by mere analysis of Li1s peak. For this reason, the sample was tilted to an emission angle of $25^{\circ}$ (to the normal of the sample surface) where it is more bulk sensitive (BS position) as demonstrated in Fig. 3a. Figure $3 \mathrm{~b}$ and c reveal the deconvoluted $\mathrm{C} 1 \mathrm{~s}$ peak at the annealing temperatures of $750{ }^{\circ} \mathrm{C}$ and $850{ }^{\circ} \mathrm{C}$ with XPS emission angle of $45^{\circ}$ (more surface sensitive) and $25^{\circ}$ (more bulk sensitive). The peak deconvolution at $750{ }^{\circ} \mathrm{C}$ reveals the presence of a diamond peak (boron doping) at $284.6 \mathrm{eV}$ [22], a peak for single bonded carbon (-C-O-) at $285.6 \mathrm{eV}$, and another peak around $283 \mathrm{eV}$ which was attributed to the metal carbide bond [36], in this case [-Li-C-]. The contribution of the metal carbide peak in bulk sensitive mode was found to be $2.04 \%$ of the total peak area, while in the surface sensitive mode it was just $0.13 \%$ of the total peak area (a difference of more than $90 \%$ ).

This is interesting as it can be true only if Li has diffused into the near-surface bulk of diamond. Zamir et al. have demonstrated the successful co-doping of $\mathrm{Li}-\mathrm{N}$ in the diamond crystal [19] using the same chemical process of Li deposition. Hence a stable interstitial doping [9] of the substrate with $\mathrm{Li}$ atoms at higher annealing temperatures is demonstrated along with the formation of stable $\mathrm{Li}-\mathrm{O}$ termination of diamond surface using the chemical route. A similar trend is seen in the case of an $850{ }^{\circ} \mathrm{C}$ annealing temperature where the peak area of $-\mathrm{M}-\mathrm{C}-$ peak in $\mathrm{BS}$ mode is more than $5 \%$ than in SS mode which shows that at $850{ }^{\circ} \mathrm{C}$ the $\mathrm{Li}$ in the bulk positions have diffused to the surface. At $850{ }^{\circ} \mathrm{C}$, the metal carbide peak in Fig. 3c can be seen to decrease in area. At $850{ }^{\circ} \mathrm{C}$, however, the significant decrease in the peak areas of $\mathrm{O} 1 s$ and Li $1 s$ in Fig. 2 a and $\mathrm{b}$ is seen which was attributed to not only the expulsion of $\mathrm{Li}$ atoms from within the near-surface bulk of diamond, but also the desorption of $\mathrm{Li}-\mathrm{O}$ groups from the surface is seen. This represents the complete dissociation of $\mathrm{C}^{+}-(\mathrm{O}-\mathrm{Li})^{-}$, which is also further strengthened by the fact that $\mathrm{C} 1 \mathrm{~s}$ peak also saturates towards $\sim 285 \mathrm{eV}$ with maximum intensity. 
Fig. 2 a $\mathrm{O} 1 s$ peaks and $\mathbf{b} \mathrm{Li}$ $1 s$ peak showing deconvoluted peaks at various annealing temperatures (a)

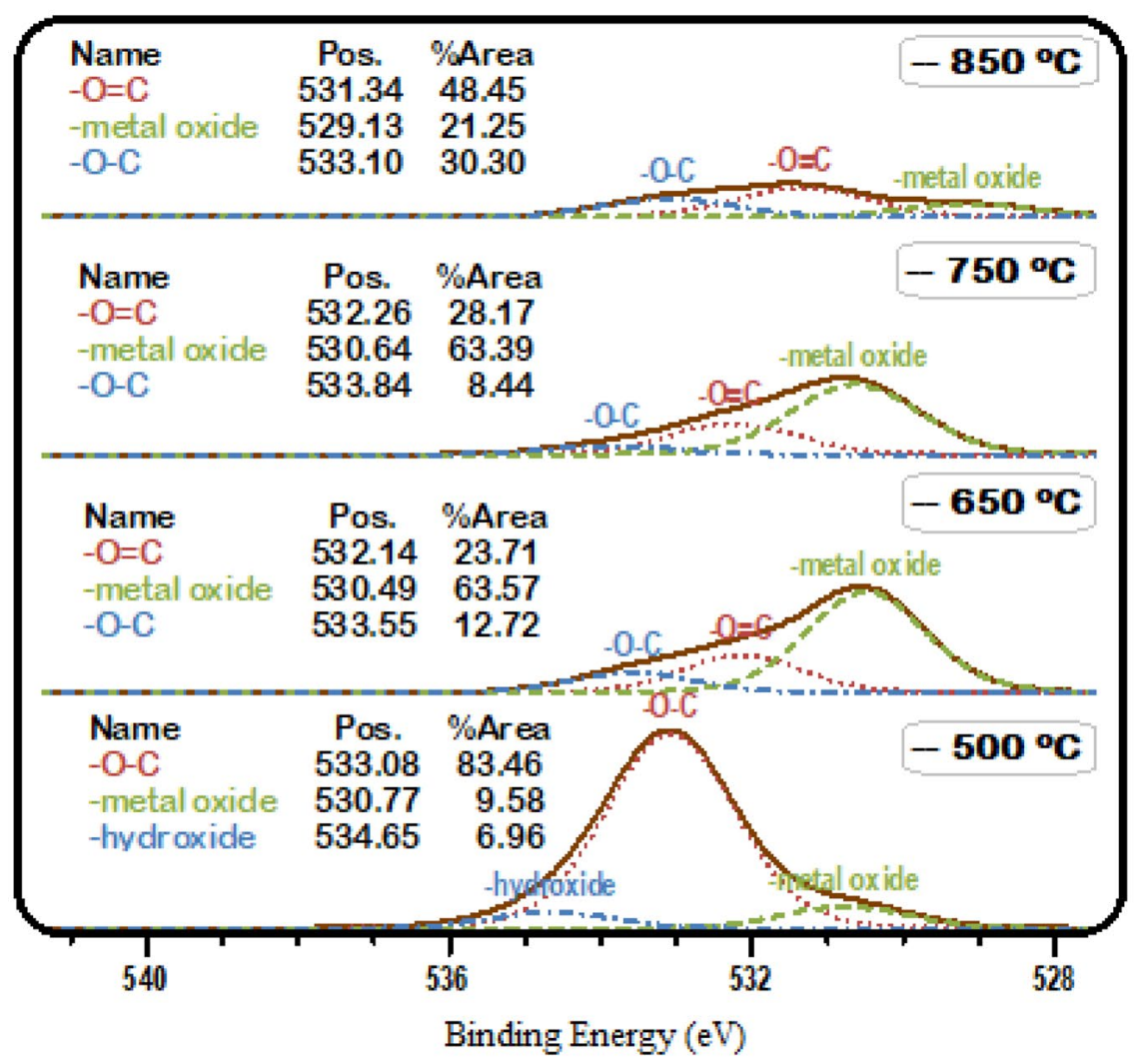

(b)

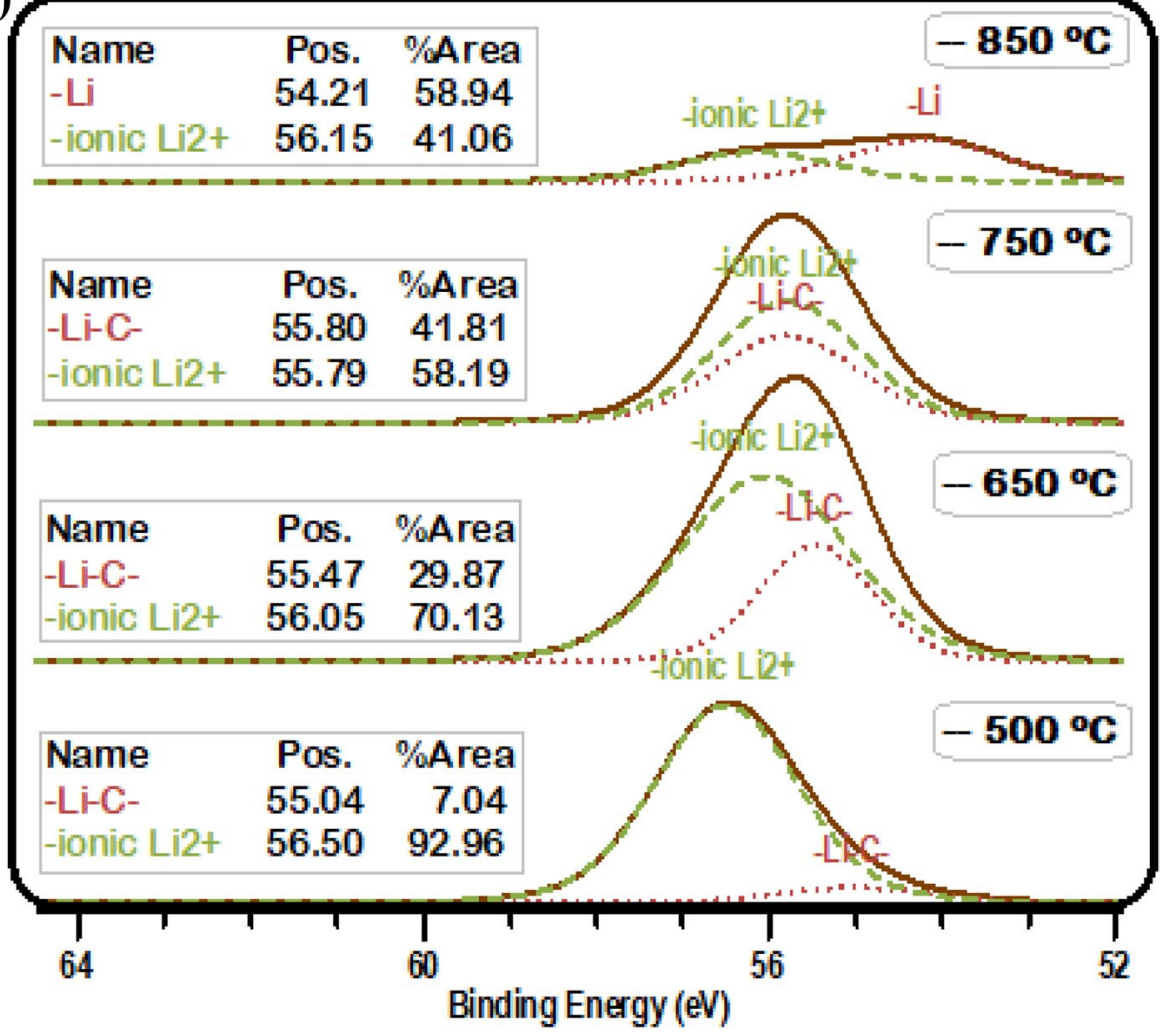


The $\mathrm{O} 1 s$ peak can also be seen again shifting towards higher binding energy with decreased intensity (expulsion of $\mathrm{O}$ and $\mathrm{Li}$ from the surface) at the same temperature, a point that will be discussed later.

The discussion on the peak areas can be summariszed by Fig. $4 \mathrm{a}$ and $\mathrm{b}$ in the form of the stoichiometric ratios between $\mathrm{O}: \mathrm{C}, \mathrm{O}: \mathrm{Li}$, and $\mathrm{C}: \mathrm{Li}$ for both samples. These were calculated by normalizing the photoemission line areas by the relative sensitivity factors and plotted at different annealing temperatures from RT to $850^{\circ} \mathrm{C}$. The bar diagram shows that as the annealing temperature is increased, the $\mathrm{Li}: \mathrm{C}$ ratio increases from 500 to $650{ }^{\circ} \mathrm{C}$ and then decreases slightly to $750{ }^{\circ} \mathrm{C}$ and then significantly at $850{ }^{\circ} \mathrm{C}$ both in surface and bulk sensitive modes. It can also be seen that $\mathrm{N}$-doped diamond shows more stable response to higher annealing temperature than B-doped polycrystalline diamond. This could be due to many grain boundaries which can affect the incorporation and diffusion of $\mathrm{Li}$ into the near-surface bulk in polycrystalline diamond than in single crystal diamond. The O:C ratio has been seen to decrease with annealing temperature, while $\mathrm{Li}$ :O first increases and then decreases beyond $750{ }^{\circ} \mathrm{C}$. A similar trend has been seen in case of nitrogen-doped HPHT diamond. An explanation for it will be given later.

Figure $5 \mathrm{~b}$ shows the B.E. shift of $\mathrm{C} 1 s, \mathrm{O} 1 s$, and the $\mathrm{C}: \mathrm{O}$ relative shift. A peak shift occurs due to the formation of surface dipole between the higher electronegative species $\mathrm{O}$ and the lower electronegative $\mathrm{C}$ atoms which result in the $\mathrm{C}+-\mathrm{O}$-dipole as demonstrated in Fig. 5a. When $\mathrm{Li}$ atoms interact with $\mathrm{O}$ it results in the transfer of more charge and hence negative potential on $\mathrm{O}$ atoms which leads to a lower B.E. as can be seen in the Figs. $2 a$ and $5 b$ where peak shifts of $\mathrm{O}$ have an upward trend. A similar trend can be seen in $\mathrm{C}$ atoms. The Li diffusion into the near-surface bulk region of diamond would increase the electric potential on the $\mathrm{C}$ atoms and hence a shift of $\mathrm{C}$ atoms towards lower B.E. (this can also be seen as an upward trend in C $1 s$ shift in Fig. 5b). A plateau was seen in case of $\mathrm{C} 1 \mathrm{~s}$ peak shift at the annealing temperature of $650^{\circ} \mathrm{C}$ which indicates a saturation point. $\mathrm{O}$ atoms reach their maximum shift at $650{ }^{\circ} \mathrm{C}$, and thereafter, the shift decreases slightly. This can be interpreted in the following way. The near-surface bulk of diamond reaches a saturation point of retaining $\mathrm{Li}$ atoms at $650{ }^{\circ} \mathrm{C}$ which continues through $750{ }^{\circ} \mathrm{C}$, while the maximum interaction occurs between $\mathrm{Li}$ and $\mathrm{O}$ atoms at the surface at $650{ }^{\circ} \mathrm{C}$ indicating the formation of a sub-monolayer of $\mathrm{LiO}_{2}$ on the surface of diamond. After $750{ }^{\circ} \mathrm{C}$, the Li atoms start coming out of the diamond bulk and at the same time $\mathrm{Li}-\mathrm{O}$ surface groups start desorbing from the surface resulting in the shift of $\mathrm{C} 1 s$ peak towards higher B.E. (a downward trend in B.E. of $\mathrm{C} 1 s$ ). Similarly, the surface-bonded $\mathrm{O}$ also shifts slightly towards higher binding energy due to Li leaving the surface along with the desorbing $\mathrm{O}$ atoms at higher annealing temperature of $850{ }^{\circ} \mathrm{C}$.
The shift and asymmetry in C $1 s$ peak of diamond surface with functionalization has been attributed to the surface band bending and surface state formation. This can be calculated using Maier's approach [37]. Using the B.E. of the $\mathrm{C} 1 \mathrm{~s}$ bulk core-level component and the fixed energy separation of $283.9 \pm 0.1 \mathrm{eV}$ between the VBM and the $\mathrm{C} 1 s$ core level, the VBM values at various annealing conditions were calculated as shown in Fig. 5c. CBM was calculated by adding experimental band gap value of $5.47 \mathrm{eV}$ to the VBM.

There is a slight upward band bending at annealing temperature of $650{ }^{\circ} \mathrm{C}$ at which saturation of $\mathrm{Li}-\mathrm{O}$ bonding and Li diffusion into near-surface diamond bulk occurs which then bends again downwards (similar to the XPS peak shift) at $850{ }^{\circ} \mathrm{C}$ due to the expulsion of $\mathrm{Li}$ atoms from diamond bulk and desorption of $\mathrm{Li}-\mathrm{O}$ groups from the surface.

A possible explanation summarizing the results obtained could be, as diagrammatically shown in Fig. 6, that initially upon annealing at $500{ }^{\circ} \mathrm{C}$, Li starts to break out of the solution and starts bonding with the oxygen which is present on the surface of the diamond. These are bonded as a mix of ether and carbonyl bonds to form a $\mathrm{LiO}_{2}$ termination on the diamond surface. Some of the $\mathrm{Li}$ atoms enter the interstitial sites inside the near-surface diamond bulk and impart negative potential to the $\mathrm{C}$ atoms. As annealing temperature increases to $650{ }^{\circ} \mathrm{C}$, the $\mathrm{Li}-\mathrm{O}$ bonding reaches saturation, and we see a change in C1s peak shift and band structure changes. The stability of Li termination on the surface of diamond and $\mathrm{Li}$ atoms inside the diamond bulk has been established before [19, 32]. No change occurs at $750{ }^{\circ} \mathrm{C}$ until the annealing temperature is further increased to $850{ }^{\circ} \mathrm{C}$ where the $\mathrm{Li}$ which is mobile inside the diamond lattice[9, 19] starts diffusing and forms -O-Li groups many of which desorb at the same temperature from the surface[38]. A chemical route of creating $\mathrm{O}-\mathrm{Li}$ termination on the surface of diamond and diffusion of $\mathrm{Li}$ atoms into the near-surface bulk was determined. This was stable up to $850^{\circ} \mathrm{C}$.

An increase in the surface conductivity can be expected with increasing Li concentration in the near-surface bulk as a shift in the valence band maximum and conduction band minimum was seen (Fig. 5c). Moreover, Li also increases the electron density near the surface of the diamond and hence changes the surface conductivity as well. This trend can be seen up to $650{ }^{\circ} \mathrm{C}$ and falls drastically at $850{ }^{\circ} \mathrm{C}$ at which point the $\mathrm{Li}$ has almost fully desorbed from the near-surface bulk of diamond as seen in Fig. 3c.

Lithium and lithium oxide termination of diamond has been shown to induce NEA on the surface of the diamond and hence decrease the work function (WF) significantly up to a temperature of $800{ }^{\circ} \mathrm{C}[32,38]$. Hence, a decrease in the WF of the diamond surface in our sample up to $750{ }^{\circ} \mathrm{C}$ is expected. Up to this temperature, $\mathrm{O}-\mathrm{Li}$ bonds are stable on the diamond surface before they desorb at $850{ }^{\circ} \mathrm{C}$. 
(a)

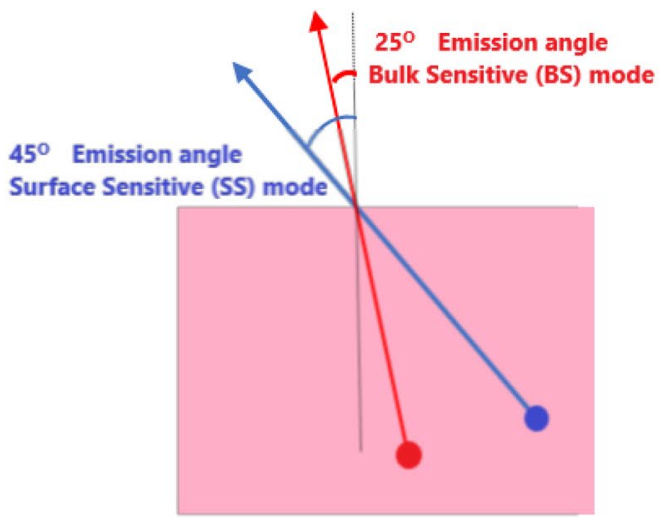

(b)

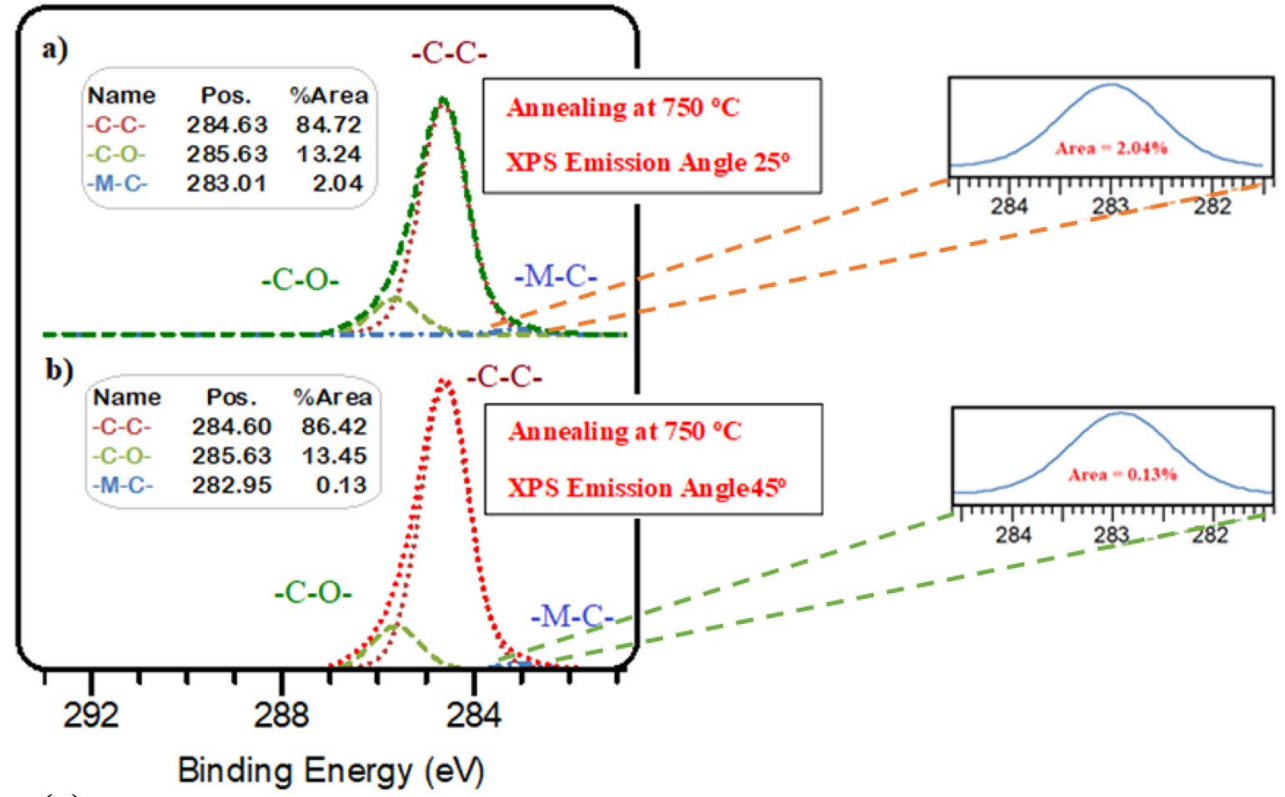

(c)

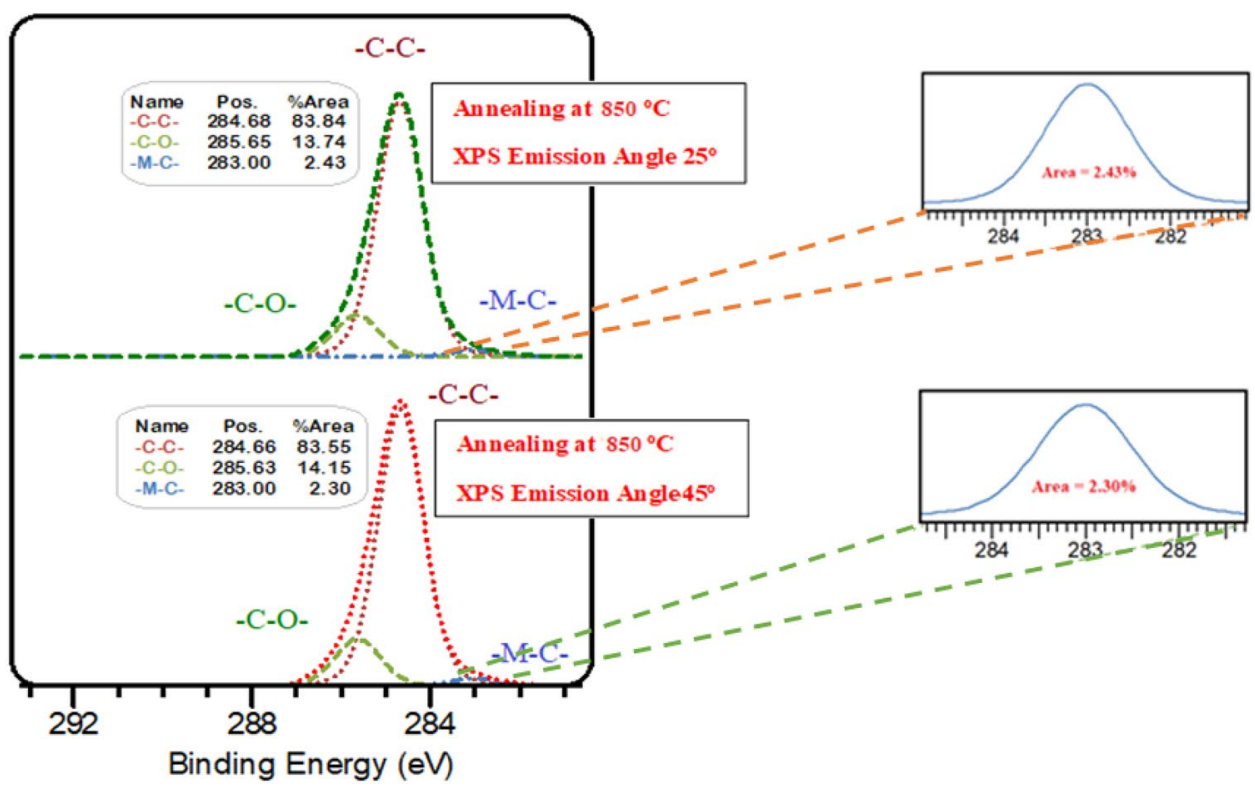


4Fig. 3 a Angle-resolved XPS showing Bulk sensitive (BS) and surface sensitive (SS) modes at $25^{\circ}$ and $45^{\circ}$ to the normal of the sample. b Angle-resolved XPS of C1s peak in the BS mode $\left(25^{\circ}\right)$ and SS mode $\left(45^{\circ}\right)$ at $750{ }^{\circ} \mathrm{C}$. c Angle-resolved XPS of C $1 s$ peak in the BS mode $\left(25^{\circ}\right)$ and $\mathrm{SS}$ mode $\left(45^{\circ}\right)$ at $850^{\circ} \mathrm{C}$

\section{Conclusion}

The interaction between $\mathrm{Li}, \mathrm{O}$, and $\mathrm{C}$ species on the surface of diamond was visualized with increasing annealing temperature. This is an important study to realize a metal oxide-terminated diamond surface for low work function thermionic devices. The possible intercalation of $\mathrm{Li}$ inside the diamond sample has also been demonstrated. The Li was shown to reach the surface of the diamond crystal as the temperature is increased to form a stable-O-Li termination up to $850{ }^{\circ} \mathrm{C}$. This is a novel and easy chemical approach of forming such terminations as previously it has been demonstrated using physical vapor deposition methods only. We have been able to prove this from the relative intensity and
Fig. 4 a The relative intensities of $\mathrm{Li}: \mathrm{C}, \mathrm{O}: \mathrm{C}$, and $\mathrm{Li}: \mathrm{O}$ ratios; for Boron-Doped polycrystalline diamond. $\mathbf{b}$ relative intensities of Li:C, O:C, and Li:O ratios for Nitrogen-Doped single crystal diamond (a)

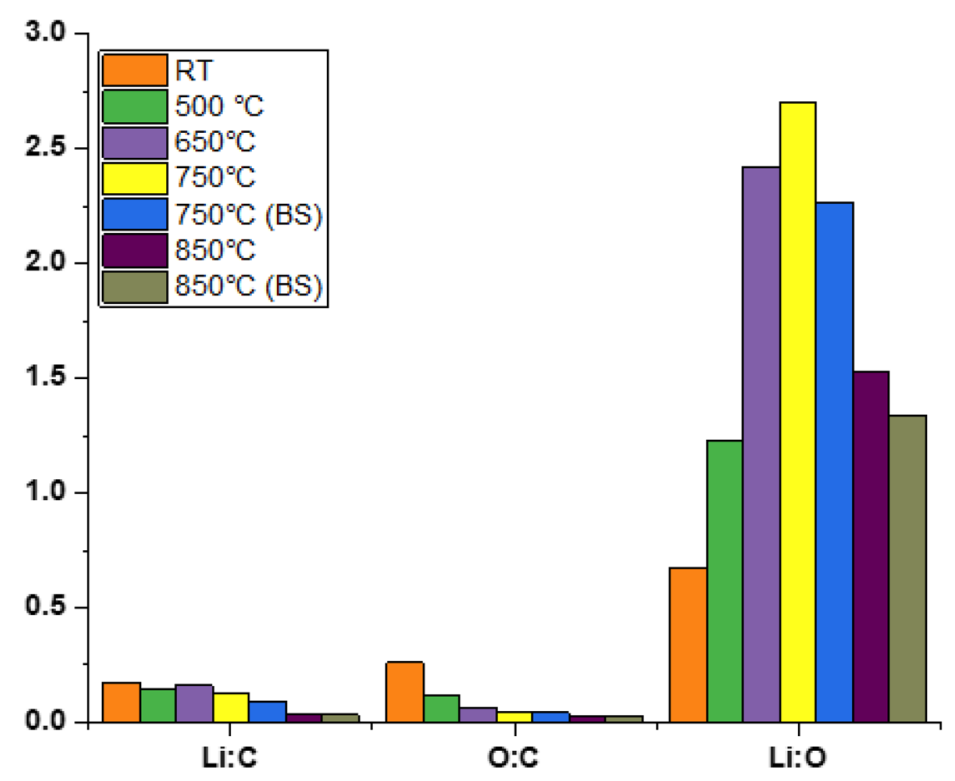

(b)

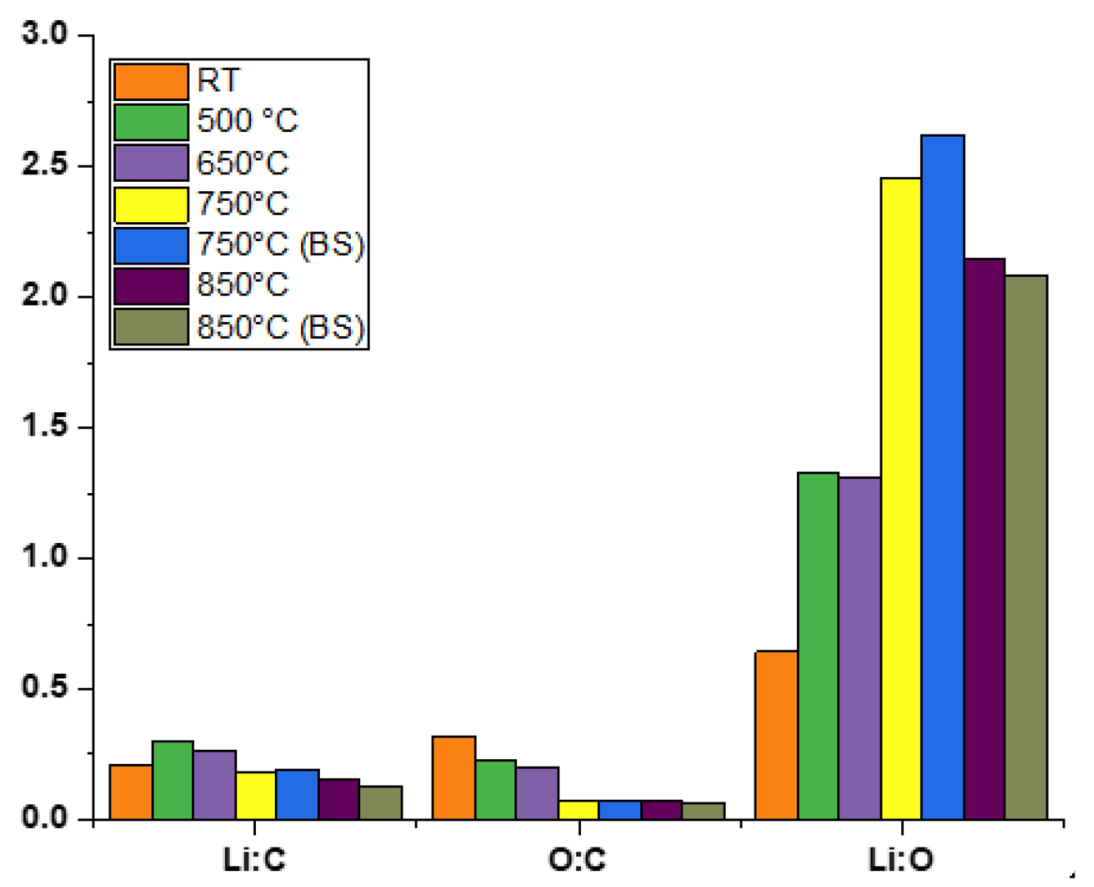


(a)

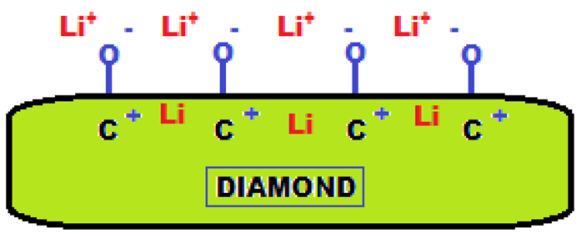

(c)

\begin{tabular}{|c|c|c|}
\hline $\mathrm{CB}$ & $\mathrm{CB}$ & $\mathrm{CB}$ \\
\hline $\mathrm{CBM}=4.72 \mathrm{eV}$ & $\mathrm{CBM}=4.77 \mathrm{eV}$ & $\mathrm{CBM}=4.72 \mathrm{eV}$ \\
$-\mathbf{C}$ & Fermi Level, $\mathrm{E}_{\mathrm{f}}=0$ & $\mathbf{-}-\mathbf{- ~ - ~ - ~}$ \\
$\mathrm{VBM}=0.75 \mathrm{eV}$ & $\mathrm{VBM}=0.7 \mathrm{eV}$ & $\mathrm{VBM}=0.75 \mathrm{eV}$ \\
\hline $\mathrm{VB}$ & $\mathrm{VB}$ & $\mathrm{VB}$ \\
\hline
\end{tabular}

(b)

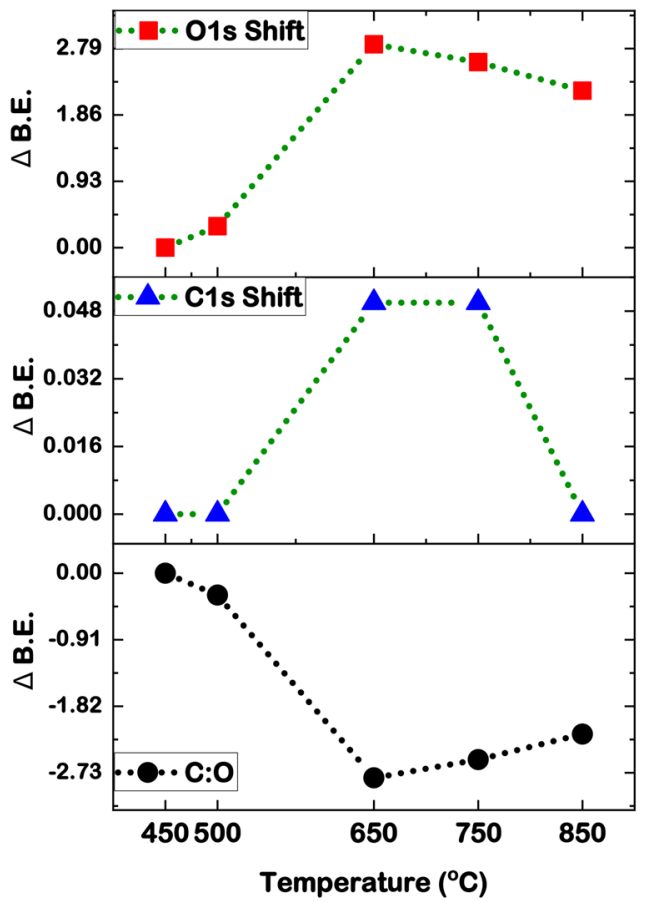

Fig. 5 a $\mathrm{O}-\mathrm{C}$ dipole representation of the diamond surface showing the dipole between $\mathrm{C}-\mathrm{O}$ due to charge transfer between the species. b $\mathrm{O} 1 s, \mathrm{C} 1 s$, and $\mathrm{C}: \mathrm{O}$ relative shifts showing a saturation point at $650{ }^{\circ} \mathrm{C}$ and significant changes at $850{ }^{\circ} \mathrm{C}$ as explained. c The band structure of diamond showing the position of Valence band maximum (VBM) and Conduction band Maximum $(\mathrm{CBM})$ at $500{ }^{\circ} \mathrm{C}, 650{ }^{\circ} \mathrm{C}$, and $850{ }^{\circ} \mathrm{C}$

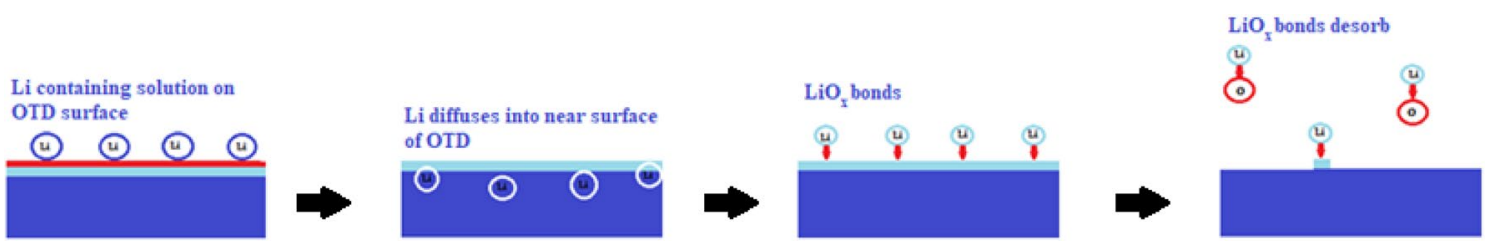

Fig. 6 The process of $\mathrm{O}-\mathrm{Li}$ adsorption and Li diffusion into the near-surface diamond bulk at the initial annealing temperatures and desorption of $\mathrm{LiO}_{x}$ groups at higher annealing temperatures

shifts of XPS peaks in BDD and Nitrogen-doped HPHT single crystal samples. This work could serve as a an insight into the alloy termination of diamond with alkali and other higher order metals, some of which have been predicted to lower the work function significantly.

Acknowledgements The authors acknowledge the Bristol NanoESCA Facility (EPSRC Strategic Equipment Grant EP/K035746/1 and EP/ M000605/1). S.U. acknowledges the Ph.D. studentship funded through BCFN: The Zutshi Smith Scholarship, University of Bristol.

Author contributions The manuscript was written through contributions of all authors. All authors have given approval to the final version of the manuscript.
Data Availability The datasets generated during and/or analysed during the current study are available from the corresponding author on reasonable request.

\section{Declarations}

Conflict of interest The authors declare no competing financial interest.

Open Access This article is licensed under a Creative Commons Attribution 4.0 International License, which permits use, sharing, adaptation, distribution and reproduction in any medium or format, as long as you give appropriate credit to the original author(s) and the source, provide a link to the Creative Commons licence, and indicate if changes were made. The images or other third party material in this article are included in the article's Creative Commons licence, unless indicated 
otherwise in a credit line to the material. If material is not included in the article's Creative Commons licence and your intended use is not permitted by statutory regulation or exceeds the permitted use, you will need to obtain permission directly from the copyright holder. To view a copy of this licence, visit http://creativecommons.org/licenses/by/4.0/.

\section{References}

1. B.C. Djubua, N.N. Chubun, Emission properties of spindt-type cold cathodes with different emission cone material. IEEE Trans. Electron Dev. 38(10), 2314-2316 (1991). https://doi.org/10.1109/ 16.88516

2. W.F. Paxton, A. Steigerwald, M. Howell, N. Tolk, W.P. Kang, J.L. Davidson, The effect of hydrogen desorption kinetics on thermionic emission from polycrystalline chemical vapor deposited diamond. Appl. Phys. Lett. (2012). https://doi.org/10.1063/1.47720 69

3. F.A.M. Koeck, R.J. Nemanich, A. Lazea, K. Haenen, Thermionic electron emission from low work-function phosphorus doped diamond films. Diam. Relat. Mater. 18(5-8), 789-791 (2009). https:// doi.org/10.1016/j.diamond.2009.01.024

4. F.A.M. Koeck, R.J. Nemanich, Sulfur doped nanocrystalline diamond films as field enhancement based thermionic emitters and their role in energy conversion. Diam. Relat. Mater. 14(11-12), 2051-2054 (2005). https://doi.org/10.1016/j.diamond.2005.09. 001

5. E.B. Lombardi, A. Mainwood, K. Osuch, Ab initio study of lithium and sodium in diamond. Phys. Rev. B (2007). https://doi.org/ 10.1103/PhysRevB.76.155203

6. N. Jiang, A. Hatta, T. Ito, Nitrogen doping effects on electrical properties of diamond films. Jpn. J. Appl. Phys. Lett. (1998). https://doi.org/10.1143/jjap.37.11175

7. J.P. Goss, R.J. Eyre, P.R. Briddon, Theoretical models for doping diamond for semiconductor applications. Phys. Status Solidi 245(9), 1679-1700 (2008). https://doi.org/10.1002/pssb.20074 4115

8. A. Mainwood, Theoretical modelling of dopants in diamond. J. Mater. Sci. 17(6), 453-458 (2006). https://doi.org/10.1007/ s10854-006-8091-x

9. S.A. Kajihara, A. Antonelli, J. Bernholc, R. Car, Nitrogen and potential n-type dopants in diamond. Phys. Rev. Lett. 66(15), 2010-2013 (1991). https://doi.org/10.1103/PhysRevLett.66.2010

10. E.B. Lombardi, A. Mainwood, A first principles study of lithium, sodium and aluminum in diamond. Diam. Relat. Mater. 17(7-10), 1349-1352 (2008). https://doi.org/10.1016/j.diamond.2007.12. 015

11. G. Popovici, R.G. Wilson, T. Sung, M.A. Prelas, S. Khasawinah, Diffusion of boron, lithium, oxygen, hydrogen, and nitrogen in type IIa natural diamond. J. Appl. Phys. 77(10), 5103-5106 (1995). https://doi.org/10.1063/1.359320

12. R. Job, M. Werner, A. Denisenko, A. Zaitsev, W.R. Fahrner, Electrical properties of lithium-implanted layers on synthetic diamond. Diam. Relat. Mater. 5(6-8), 757-760 (1996). https://doi.org/10. 1016/0925-9635(95)00458-0

13. M. Restle et al., Lattice sites of ion implanted $\mathrm{Li}$ in diamond. Appl. Phys. Lett. 66(20), 2733-2735 (1995). https://doi.org/10. 1063/1.113691

14. C. Uzan-Saguy, C. Cytermann, B. Fizgeer, V. Richter, R. Brener, R. Kalish, Diffusion of lithium in diamond. Physica Status Solidi (A) Appl. Res. 193(3), 508-516 (2002)

15. G. Popovici, T. Sung, S. Khasawinah, M.A. Prelas, R.G. Wilson, Forced diffusion of impurities in natural diamond and polycrystalline diamond films. J. Appl. Phys. 77(11), 5625-5629 (1995). https://doi.org/10.1063/1.359204

16. K. Okumura, J. Mort, M. Machonkin, Lithium doping and photoemission of diamond thin films. Appl. Phys. Lett. 57(18), 19071909 (1990). https://doi.org/10.1063/1.104008

17. R. Zeisel, C.E. Nebel, M. Stutzmann, H. Sternschulte, M. Schreck, B. Stritzker, Photoconductivity study of Li doped homoepitaxially grown CVD diamond. Phys. Status Solidi Appl. Res. 181(1), 45-50 (2000). https://doi.org/10.1002/1521-396X(200009) 181:1\%3c45::AID-PSSA45\%3e3.0.CO;2-2

18. H. Sachdev, R. Haubner, B. Lux, Lithium addition during CVD diamond deposition using lithium tert.-butanolat as precursor. Diam. Relat. Mater. 6(2-4), 494-500 (1997). https://doi.org/10. 1016/s0925-9635(96)00628-0

19. M.Z. Othman, P.W. May, N.A. Fox, P.J. Heard, Incorporation of lithium and nitrogen into CVD diamond thin films. Diam. Relat. Mater. 44, 1-7 (2014). https://doi.org/10.1016/J.DIAMOND. 2014.02.001

20. L. Diederich, P. Aebi, O.M. Küttel, L. Schlapbach, NEA peak of the differently terminated and oriented diamond surfaces. Surf. Sci. (1999). https://doi.org/10.1016/S0039-6028(99)00210-1

21. L. Diederich, O.M. Küttel, P. Aebi, E. Maillard-Schaller, R. Fasel, L. Schlapbach, Photoelectron emission from the negative electron affinity caesiated natural diamond (100) surface. Diam. Relat. Mater. 7(2-5), 660-665 (1998). https://doi.org/10.1016/ s0925-9635(97)00284-7

22. G. Wan, M. Cattelan, N.A. Fox, Electronic structure tunability of diamonds by surface functionalization. J. Phys. Chem. C (2019). https://doi.org/10.1021/acs.jpcc.8b11232

23. F. Maier, M. Riedel, B. Mantel, J. Ristein, L. Ley, Origin of surface conductivity in diamond. Phys. Rev. Lett. 85(16), 3472 (2000)

24. D. Takeuchi, M. Riedel, J. Ristein, L. Ley, Surface band bending and surface conductivity of hydrogenated diamond. Phys. Rev. B (2003). https://doi.org/10.1103/PhysRevB.68.041304

25. M.C. James, P.W. May, N.L. Allan, Ab initio study of negative electron affinity from light metals on the oxygen-terminated diamond (1 11 1) surface. J. Phys. Condens. Matter 31(29), 295002 (2019). https://doi.org/10.1088/1361-648X/ab18ef

26. K.P. Loh, J.S. Foord, R.G. Egdell, R.B. Jackman, Tuning the electron affinity of CVD diamond with adsorbed caesium and oxygen layers. Diam. Relat. Mater. 6(5-7), 874-878 (1997). https://doi. org/10.1016/s0925-9635(96)00737-6

27. P.K. Baumann, R.J. Nemanich, Electron affinity and Schottky barrier height of metal-diamond (100), (111), and (110) interfaces. J. Appl. Phys. 83(4), 2072-2082 (1998). https://doi.org/ 10.1063/1.366940

28. S. Petrick, C. Benndorf, Potassium adsorption on hydrogenand oxygen-terminated diamond (100) surfaces. Diam. Relat. Mater. 10(3-7), 519-525 (2001). https://doi.org/10.1016/S09259635(00)00440-4

29. K.W. Wong, Y.M. Wang, S.T. Lee, R.W.M. Kwok, Negative electron affinity on polycrystalline diamond surface induced by lithium fluoride deposition. Diam. Relat. Mater. 8(10), 18851890 (1999). https://doi.org/10.1016/S0925-9635(99)00149-1

30. K.W. Wong, Y.M. Wang, S.T. Lee, R.W.M. Kwok, Lowering of work function induced by deposition of ultra-thin rubidium fluoride layer on polycrystalline diamond surface. Appl. Surf. Sci. 140(1-2), 144-149 (1999). https://doi.org/10.1016/S01694332(98)00582-0

31. K.M. O'Donnell, T.L. Martin, N.A. Fox, D. Cherns, Ab initio investigation of lithium on the diamond C(100) surface. Phys. Rev. B (2010). https://doi.org/10.1103/PhysRevB.82.115303

32. K.M. O'Donnell et al., Diamond surfaces with air-stable negative electron affinity and giant electron yield enhancement. Adv. Funct. 
Mater. 23(45), 5608-5614 (2013). https://doi.org/10.1002/adfm. 201301424

33. P.W. May, J.C. Stone, M.N.R. Ashfold, K.R. Hallam, W.N. Wang, N.A. Fox, The effect of diamond surface termination species upon field emission properties. Diam. Relat. Mater. 7(2-5), 671-676 (1998). https://doi.org/10.1016/s0925-9635(97)00181-7

34. J.B. Cui, J. Ristein, M. Stammler, K. Janischowsky, G. Kleber, L. Ley, Hydrogen termination and electron emission from CVD diamond surfaces: a combined secondary electron emission, photoelectron emission microscopy, photoelectron yield, and field emission study. Diam. Relat. Mater. 9(3), 1143-1147 (2000). https:// doi.org/10.1016/S0925-9635(99)00279-4
35. J.P. Contour, A. Salesse, M. Froment, M. Garreau, J. Thevenin, J. Microsc. Spectrosc. Electron. 4, 483 (2000)

36. A.V. Shchukarev, D.V. Korolkov, XPS study of group IA carbonates. Cent. Eur. J. Chem. 2(2), 347-362 (2004). https://doi.org/10. 2478/BF02475578

37. F. Maier, J. Ristein, L. Ley, Electron affinity of plasma-hydrogenated and chemically oxidized diamond (100) surfaces. Phys. Rev. B 64(16), 165411 (2001). https://doi.org/10.1103/PhysRevB.64. 165411

38. K.M. O'Donnell et al., Photoelectron emission from lithiated diamond. Phys. Status Solidi Appl. Mater. Sci. 211(10), 2209-2222 (2014). https://doi.org/10.1002/pssa.201431414 